\title{
A Study of Parental Acceptance - Rejection and Aggression Towards Parents of Adolescence
}

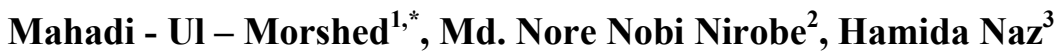 \\ Department of Psychology, Jagannath University, Bangladesh
}

Copyright (C) 2015 by authors, all rights reserved. Authors agree that this article remains permanently open access under the terms of the Creative Commons Attribution License 4.0 International License

\begin{abstract}
The aim of the present study was to investigate whether there is any significant relation between parental acceptance - rejection and aggression towards parents of adolescence. For this purpose 960 students were selected randomly from various institution of Dhaka city. Among of them 934 students respond for investigation and used following questionnaire - a. Adult PARQ: Father \& Mother Version of Rohner[11], B. Aggression Towards Parents of Dolan[5]. In this study it is found that, the significant positive correlation between aggression towards parents and sub parts of parental acceptance-rejection(Father warmth /affection, neglect/indifference, rejection/undifference, Mother warmth/affection, Mother control and Mother total) and there is no significant difference between male and female according to aggression towards parents. This result implies that, there is an effect on parent child interaction.
\end{abstract}

Keywords Parental Acceptance - Rejection \& Aggression Towards Parents

\section{Introduction}

Adolescent aggression towards parents is an issue that has been traditionally neglected in social research and, in fact, no direct studies on the topic appears to have been undertaken in most European countries. There is only few available data based on surveys mainly conducted in North America. Parents often show a profound rejection to reporting violence of their children, which makes it impossible to know the real number of incidents of parental abuse. Mothers and fathers show a great tendency to deny the seriousness of the aggressive attacks in order to perpetuate the myth of family harmony (Harbin and Madden [6]. Protectiveness and secretiveness about the family situation represents a way of protecting their self-image as parents (Pagani ., Larocque ., Vitaro, and Tremblay [8]. Parental shame, fear of blame, and community judgment of their capacity to parent are factors that contribute to parents maintaining secrecy (Bobic, [3] and (Agnew and Huguley [1]. A feeling of loneliness and lack of social support, as well as the belief of failure on parenting is common among these parents; moreover these factors increase stress within the family which worsen the situation. Furthermore, figures available hitherto must be cautiously interpreted and cannot be generalized since cultural background may exert a remarkable influence on them. Not only comparisons of general prevalence are difficult, but also comparisons of types of violence exerted against parents, since most of research was conducted in the 1980s and early 1990s, when only physical aggression was assessed while psychological, emotional and financial abuse were completely forgotten (Bobic [2]. Every person has experienced the warmth and affection provided by someone important to him/her, who is called the parent not necessarily, mother and father. This warmth and affection is a range from a great deal to none, where one end is named parental acceptance while the other one is termed rejection (Rohner [12]. The term parent is defined in PARTheory (Rohner., Khaleque., Riaz., Khan., Sadeque., \& Laukkala [15] as any person who has a more or less long term care giving responsibility for a child. Such person may be biological or adoptive parents, older sibilngs, grandparents, other relatives or even non-kinspersons. Parental acceptance-rejection refers in PART theory to warmth dimension of parenting, with parental acceptance at the positive end of the continuum and parental rejection at the negative end. Parental acceptance refers to the love, affection, care, comfort, support, nurturance, and other such behaviors that parents can feel or express toward their children. Parental rejection refers to the absence or withdrawal of warmth, love, support, or affection by parents toward their children. Worldwide, parental acceptance-rejection can be expressed or perceived in any one or a combination of four major ways (Rohner [9] and (Rohner [10]. These include (a) warmth/affection (or its opposite, coldness/lack of affection), (b) hostility/aggression, (c) indifference/neglect, and (d) undifferentiated rejection. Undifferentiated rejection refers to individuals' belief that their parents do (or did) not really love, want, appreciate, or care about them, without necessarily experiencing any clear behavioral indicators that the parents are (or were) neglecting, unaffectionate, or aggressive toward them. 
The research program was initiated almost five decades ago in response to claims by Western social scientists that parental love is essential to the healthy social and emotional development of children. After more than two thousand studies, many inspired directly by parental acceptance-rejection theory (PAR Theory) described here at least one conclusion is clear: Children everywhere need a specific form of positive response--acceptance--from parents and other attachment figures.

When this need is not met satisfactorily, children worldwide - regardless of variations in culture, gender, age, ethnicity, or other such defining conditions-tend to report themselves to be hostile and aggressive; dependent or defensively independent, over impaired in self-esteem and self-adequacy; emotionally unresponsive; emotionally unstable; and to have a negative worldview, among other responses. Additionally, youths and adults who perceive themselves to be rejected appear to be anxious and insecure, as well as to be disposed toward behavior problems and conduct disorders, to be depressed or have depressed affect, and to become involved in drug and alcohol abuse, among other problems.

Research question: Is there any relation of parental acceptance-rejection with aggression towards parents?

\section{Rationale of the Study}

Parental acceptance and rejection is very important issue of our life. Scientific study of parental acceptance and rejection is important because it can provide positive contribution of the parents. So it is necessary to know how parental acceptance - rejection is related with aggression towards parents. The findings of this study will helpful to enrich our knowledge and will play an important role for the better understanding of parents-child relationship.

\section{Objectives of the study}

The objectives of the study are :

- To see whether there is any significant relation between parental acceptance-rejection and aggression towards Parents.

- To see whether there is any significant difference of aggression towards parents according to gender.

\section{Method}

\section{Target population}

The target population of this study was adolescence those who are going to school and college in Dhaka city.

\section{Sample and Sampling technique}

In this study students were selected randomly as a sample those who are adolescence from different educational institution in Dhaka city in Bangladesh. Generally there are three categories in education stream in Bangladesh as Bangla, English \& Madrasha medium. The lists of all educational institutions name were collected from Bangladesh Bureau of Educational Information and Statistics (BANBEIS). 4 institutions from each category in total 12 institutions were selected through lottery. In Bangladeshi cultural context, a person's adolescent period goes (13 to before 18years old) through $9,10,11 \& 12$ classes. The list of student's class ID of each class collected through school authorities. Then boys and girls were separated according to their gender. After that, ten girls and ten boys were selected according to their class ID through lottery. Each institution contains 80 students as a sample and each category contains 320 students as a sample. Thus we collected another two categories and total 960 respondents were selected from them and 934 responds $(97.30 \%)$ for investigation.

\section{Measuring Instruments}

In addition to these questionnaires a personal information form (PIF) was used to collect information about age, gender, education, occupation, father's education and profession, mother's education and profession, longevity of the intimate relationships and similar matters.

\section{Parental Acceptance - Rejection Questionnaire}

Adult Version of the Parental
Acceptance-Rejection/Control Questionnaire for Father and Mother (Adult PARQ/Control: Father \& Mother): The father and mother versions (Rohner [11] of the PARQ/control are self-report and four point Likert-type measures consisting of 73 items designed to assess individuals' perceptions of paternal and maternal acceptance or rejection and control. In both questionnaires, respondents are asked to reflect on their fathers' or mothers' behavior towards them when they were children growing up at home. Each version consists of five sub-scales. Four sub-scales (60 items) measure respondents' perceptions of paternal or maternal warmth/affection, hostility/aggression, indifference/neglect, and undifferentiated rejection. The fifth scale (13 items) measures perceived paternal or maternal behavioral control. Parental accepatnace-rejection questionnaires (Father and mother versions) were adapted in Bangla (Jasmin and Uddin [7]. Split-half reliability coefficients of adult PARQ/Control for father and mother were found 0.94, 0.89 and 0.85, respectively. Cronbach alpha coefficients of these three scales were found $0.95,0.92$ and 0.93 , respectively.

\section{Aggression Towards Parents}

This scale measures the frequency with which peers or friends shout,curse or hit their parents; or are recipients of these behaviors from their parents.Questions are asked during a one-on-one interview. This scale was developed by (Dolan [5] and adapted by ( Church[4]. Reliability/Validity: Internal Consistency not assessed. This scale was translated into Bangla by the researchers themselves.

\section{Procedure}

Random technique was followed to collect the data from 
the students of the various School, College and Madrasha of Dhaka city. They were informed of the purpose of the present study and necessary rapport was established before administering the questionnaires. The respondents were instructed to read the items of the scales attentively and to respond rapidly in PAR questionnaire not in the way they liked their father to be but how they perceived their father. As similar way, the respondents were instructed to read the items of the scales attentively and to respond rapidly in PAR questionnaire and Aggression towards Parents questionnaire. They were asked to give tick $(\sqrt{ })$ mark in the appropriate box. They were also requested not to omit any item in the questionnaire and told that there was no right or wrong answer. They were assured that, the data would be kept confidential and would be used only for research purpose. The respondents did not show any resentment. After finishing the job they were given lots of thanks. To administer all the instruments approximate average time required were 30 to 35 minutes per individual respondent. All data were collected within Four months from July, 2013 to November, 2013.

\section{Result}

Table 1. Pearson product moment correlation between Aggression towards parents and PAR Father/Mother

\begin{tabular}{|c|c|}
\hline & Aggression towards parents \\
\hline PAR Father Warmth/Affection & $.012^{*}$ \\
\hline PAR Father Aggression/Hostile & -.020 \\
\hline PAR Father Neglect/Indifference & $.007 * *$ \\
\hline PAR Father Rejection/Undifference & $.033^{*}$ \\
\hline PAR Father Control & -.045 \\
\hline PAR Father Total & -.014 \\
\hline PAR Mother Warmth/Affection & $.007 * *$ \\
\hline PAR Mother Aggression/Hostile & .056 \\
\hline PAR Mother Neglect/Indifference & -.006 \\
\hline PAR Mother Rejection/Undifference & -.013 \\
\hline PAR Mother Control & $.036^{*}$ \\
\hline PAR Mother Total & $.037 *$ \\
\hline
\end{tabular}

$* * \mathrm{P}<0.01 \quad * \mathrm{P}<0.05$

From the above table 1 indicate that the correlation coefficient between complete PAR Father version and aggression towards parents is not significant at 0.05 level and PAR Father aggression/ hostile and aggression towards parents is not significant at 0.05 level and PAR Father neglect/ indifference and aggression towards parents; is not significant at 0.01 level. PAR Father rejection/ undifference and aggression towards parents is significant at 0.05 level PAR Father control and aggression towards parents is not significant at 0.05 level; On the other hand, PAR Mother total and aggression towards parents is significant at 0.01 level and . PAR Mother Warmth/ affection and aggression towards parents is significant at 0.01 level .PAR Mother aggression/ hostile and aggression towards parents is not significant at 0.05 level. PAR Mother neglect/indifference and aggression towards parents, is not significant at 0.01 level. PAR Mother rejection/undifference and aggression towards parents, is not significant at 0.05 level. PAR Mother Control and aggression towards parents is significant at 0.05 level.

Table 2. Mean difference between male and female according to Aggression towards parents

\begin{tabular}{|c|c|c|c|c|c|c|}
\hline & Gender & $\mathrm{M}$ & $\mathrm{SD}$ & $\mathrm{t}$ & $\mathrm{df}$ & $\mathrm{Sig}$ \\
\hline $\begin{array}{c}\text { Aggression } \\
\text { towards } \\
\text { parents }\end{array}$ & Male & 6.868 & 3.366 & \multirow{2}{*}{1.515} & 932 & .130 \\
\cline { 2 - 5 } & Female & 6.521 & 3.3622 & & \\
\hline
\end{tabular}

As shown in the table 2, the mean scores of male and female are approximately equal. The $t$ value which is indicates that, there is no significant difference between male and female.

\section{Discussion}

The objectives of the present study are - to see whether there is any significant relation between parental acceptance-rejection and aggression towards parents and to see whether there is any significant difference of aggression towards parents according to gender.

On the other hand, Complete PAR Mother version and aggression towards parents is significant. PAR Mother warmth/affection and aggression towards parents is significant. PAR Mother aggression/hostile and aggression towards parents is not significant; PAR Mother neglect/indifference and aggression towards parents is not significant. PAR Mother rejection/undifference and aggression towards parents is significant. PAR Mother Control and aggression towards parents is significant.

As shown in the table 2, the mean scores of male and female are approximately equal. The $t$ value is indicates that there is no significant difference between male and female according to aggression towards parents. It is said that, if parental neglect or rejection increase then aggressive behavior of children also increase.

The present findings of this study are congruent with the existing of PAR Theory (Rohner [14]. One of the subtheory of PAR Theory assumes that, the emotional need of positive response from parents (or significant others) is a powerful motivation in children. When this need is not adequately met by attachment figures, children are predisposed emotionally and behaviorally to respond in specific ways. In particular, the subtheory postulates that rejected children are likely to feel anxious and insecure. Additionally, parental rejection is expected to lead to other personality outcomes in children and adults including: hostility, aggression, passive aggression, or problems with the management of hostility and aggression; dependence or defensive independence, depending on the form, frequency, and intensity of rejection; impaired self-esteem; impaired 
self-adequacy; emotional unresponsiveness; emotional instability; and negative worldview. According to PAR Theory, rejected persons are likely to develop a negative worldview characterized by the belief that people and the world in general are hostile, treacherous, threatening, or negative in some other way. Negative worldview, negative self-esteem, negative self-adequacy, and some of the other personality dispositions described above form the basis of mental representations or social cognitions of rejected people. This parental acceptance-rejection has wide impact on children mental health issues, such as: depression and depressed affect; behavioral problems, including conduct disorders, externalizing behaviors, and delinquency; and, substance abuse (Rohner and Britner [13]. It is an important issue for parents to show positive attitude towards their children and should concern about their positive parenting.

\section{REFERENCES}

[1] Agnew, R. \& Huguley, S. (1989). Adolescent violence towards parents. Journal of Marriage and the Family, 51, 699-711.

[2] Bobic, N. (2002). Adolescent violence towards parents: Myths and realities. Marrickville, NSW: Rosemount Youth and Family Services.

[3] Bobic, N. (2004). Adolescent aggression towards parents. Australian Domestic and Family Violence Clearinghouse. Retrieved from (2008), http://www.austdvclaringhouse.unsw.edu.au/PDF\%20files/a dolescent_violence.pdf.

[4] Church, A. T. (1994). Relating the Tellegen and five-factor models of personality structure. Journal of Personality and Social Psychology, 67, 898-909.

[5] Dolan S. (1989) Doctoral Dissertation (unpublished) University of Chicago, Department of Psychology. Chicago, IL.
[6] Harbin, H. T., \& Madden, D. J. (1979). Battered parents: A new syndrome. American Journal Psychiatry, 136, $1288-1291$

[7] Jasmin, U. H., \& Uddin, M. K. (2007). Perceived parental acceptance and psychological adjustment of Dhaka University students. The Dhaka University Journal of Psychology, 31, 93-102.

[8] Pagani, L. S., Larocque, D., Vitaro, F. \& Tremblay, R. E. (2003). Verbal and physical abuse toward mothers: The role of family configuration, environment, and coping strategies. Journal of Youth and Adolescence, 32, 215-223.8

[9] Rohner, R P. (1980). Handbook for the study of parental acceptance and rejection. Center for the Study of Parental Acceptance and Rejection, University of Connecticut, 1980; revised 1984, 1990. Second printing,1991.

[10] Rohner, R. P. (1986). The warmth dimension. Foundations of parental acceptance-rejection theory. Newbury Park, CA: Sage Publications, Inc.

[11] Rohner, R. P. (1990). Handbook for the study of parental acceptance and rejection. Storrs, CT: Rohner Research Publications.

[12] Rohner, R. P. (2000). Introduction, The Warmth Dimension, foundation of parental acceptance-rejection theory. Storrs, CT: Rohner Research Publications, pp.14, 19.

[13] Rohner, R. P., \& Britner, P. A. (2002). Worldwide mental health correlates of parental acceptance-rejection: Review of cross-cultural and intracultural evidence. Cross-Cultural Research, 36, 16-47.

[14] Rohner, R. P. (2002). Parental acceptance-rejection bibliography. Retrieved from vm.uconn.edu/ rohner.

[15] Rohner, R. P., Khaleque, A., Riaz, M. N., Khan, U., Sadeque, S., \& Laukkala, H. (2005). Agreement between children's and mothers' perceptions of maternal acceptance and rejection: A comparative study of Finland and Pakistan. Ethos, 33, 299-334. 